\title{
Combining phonon accuracy with high transferability in Gaussian approximation potential models
}

Cite as: J. Chem. Phys. 153, 044104 (2020); https://doi.org/10.1063/5.0013826

Submitted: 14 May 2020 . Accepted: 02 July 2020 . Published Online: 23 July 2020

(iD) Janine George, (D) Geoffroy Hautier, (iD) Albert P. Bartók, (D) Gábor Csányi, and (iD) Volker L. Deringer

\section{COLLECTIONS}

Paper published as part of the special topic on Machine Learning Meets Chemical PhysicsMACH2O2O
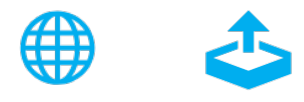

\section{ARTICLES YOU MAY BE INTERESTED IN}

An accurate and transferable machine learning potential for carbon

The Journal of Chemical Physics 153, 034702 (2020); https://doi.org/10.1063/5.0005084

Machine learning for interatomic potential models

The Journal of Chemical Physics 152, 050902 (2020); https://doi.org/10.1063/1.5126336

Hierarchical machine learning of potential energy surfaces

The Journal of Chemical Physics 152, 204110 (2020); https://doi.org/10.1063/5.0006498

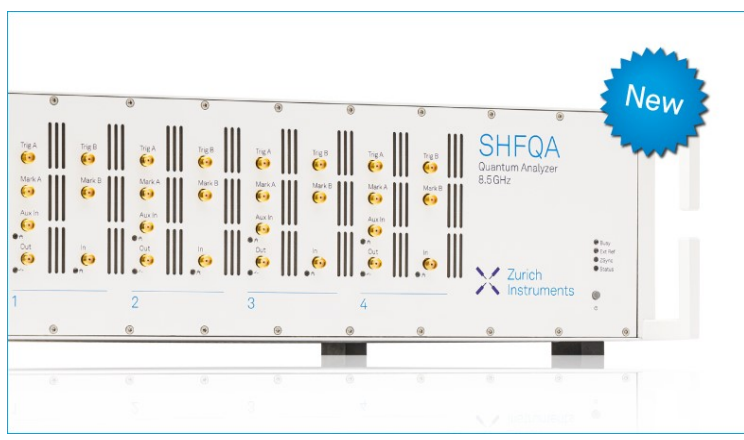

\section{Your Qubits. Measured.}

Meet the next generation of quantum analyzers

- Readout for up to 64 qubits

- Operation at up to $8.5 \mathrm{GHz}$,

Find out more

- Signal optimization with minimal latency 


\title{
Combining phonon accuracy with high transferability in Gaussian approximation potential models
}

\author{
Cite as: J. Chem. Phys. 153, 044104 (2020); doi: 10.1063/5.0013826 \\ Submitted: 14 May 2020 - Accepted: 2 July 2020 • \\ Published Online: 23 July 2020
}

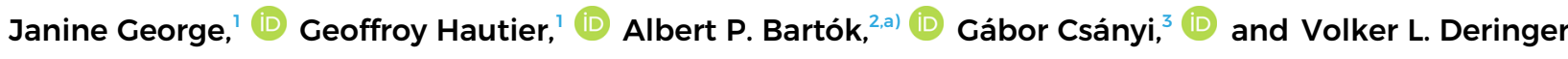

AFFILIATIONS

${ }^{1}$ Institute of Condensed Matter and Nanosciences, Université catholique de Louvain, Chemin des Étoiles 8 ,

1348 Louvain-la-Neuve, Belgium

${ }^{2}$ Department of Physics and Warwick Centre for Predictive Modelling, School of Engineering, University of Warwick, Coventry CV4 7AL, United Kingdom

${ }^{3}$ Engineering Laboratory, University of Cambridge, Cambridge CB2 1PZ, United Kingdom

${ }^{4}$ Department of Chemistry, Inorganic Chemistry Laboratory, University of Oxford, Oxford OX1 3QR, United Kingdom
\end{abstract}

Note: This paper is part of the JCP Special Topic on Machine Learning Meets Chemical Physics.

also at: Rutherford Appleton Laboratory, Scientific Computing Department, Science and Technology Facilities Council,

Didcot, OX11 OQX, United Kingdom

b) Author to whom correspondence should be addressed:volker.deringer@chem.ox.ac.uk

\begin{abstract}
Machine learning driven interatomic potentials, including Gaussian approximation potential (GAP) models, are emerging tools for atomistic simulations. Here, we address the methodological question of how one can fit GAP models that accurately predict vibrational properties in specific regions of configuration space while retaining flexibility and transferability to others. We use an adaptive regularization of the GAP fit that scales with the absolute force magnitude on any given atom, thereby exploring the Bayesian interpretation of GAP regularization as an "expected error" and its impact on the prediction of physical properties for a material of interest. The approach enables excellent predictions of phonon modes (to within $0.1 \mathrm{THz}-0.2 \mathrm{THz}$ ) for structurally diverse silicon allotropes, and it can be coupled with existing fitting databases for high transferability across different regions of configuration space, which we demonstrate for liquid and amorphous silicon. These findings and workflows are expected to be useful for GAP-driven materials modeling more generally.
\end{abstract}

Published under license by AIP Publishing. https://doi.org/10.1063/5.0013826

\section{INTRODUCTION}

Vibrational properties on the atomic scale determine the thermal behavior of materials. Their knowledge is therefore of central importance in many fields of physics, materials science, and engineering. For example, a low thermal conductivity is a requirement for thermoelectric waste-heat recovery, ${ }^{1}$ and the ability to predict this property based on highly accurate theoretical and computational methods can allow the community to discover possible, new thermoelectric materials. Indeed, thermal conductivity and other macroscopic quantities can nowadays be computed from first principles, normally based on density-functional theory (DFT), although at a very substantial computational cost. $^{2-7}$ Only very recently, larger DFT-computed databases of harmonic vibrational properties became available, ${ }^{8,9}$ but high-throughput predictions of more computationally expensive properties such as thermal conductivities have not been attempted to our knowledge. So far, computational searches for materials with low thermal conductivity have been performed based on computationally cheaper models such as the quasiharmonic Debye model, which do not require the full ab initio computation of the thermal conductivity, or those combined with global optimization techniques, which only require a limited amount of full $a b$ initio computations of the thermal conductivity. ${ }^{10,1}$

In an effort to sidestep the computational cost of DFT, machine learning (ML) based interatomic potential models are increasingly and widely used in materials modeling. ${ }^{12-15}$ Based on a reference 
database of (typically) DFT data and a regression framework including artificial neural networks, ${ }^{16-19}$ kernel methods, ${ }^{20-22}$ or linear fitting, ${ }^{23-25}$ they enable atomistic simulations at similar accuracy levels but at orders of magnitude lower computational cost. The high accuracy that ML potentials can reach for phonons was demonstrated a decade ago already, ${ }^{26}$ and early applications to amorphous phases showcased the ability to treat large and structurally complex systems. ${ }^{27,28}$ To date, vibrational properties continue to be a sensitive test for the quality of a candidate potential ${ }^{29-32}$ because they give a direct and physically meaningful measure of how reliably the interatomic forces are predicted by any ML model. Notably, a recent benchmark study has shown that a range of different ML potential fitting frameworks can lead to phonon predictions in excellent agreement with DFT reference data.

Beyond the prediction of harmonic phonons in crystals, ML potentials have begun to be used for thermal properties including anharmonic effects as well. A proof-of-concept in the Gaussian approximation potential (GAP) framework, for the temperaturedependent phonon dispersion curves of $\mathrm{Zr}$, was reported in $2018^{3}$ Skutterudite $\mathrm{CoSb}_{3}$ (Ref. 34) and elemental metals ${ }^{35}$ were studied using Moment Tensor Potentials (MTPs) very recently, showing excellent agreement with DFT reference values. Three independent studies dealt explicitly with crystalline diamond-type (dia) silicon. $^{36-38}$

An aspect that so far has been rarely discussed in this context is the ability of materials to crystallize in different structures ("allotropes" for elements, "polymorphs" for compounds). The evaluation of phonon properties of silicon with ML potentials has focused, so far, almost exclusively on the most abundant structure, viz., the dia allotrope. ${ }^{36-38}$ There is, however, ample interest in others: clathrate-type structures have been extensively studied by DFT; ${ }^{39-41}$ many other possible silicon allotropes have been proposed. ${ }^{42-47}$ An open-framework structure, oS24, was synthesized by de-intercalation from $\mathrm{Na}_{4} \mathrm{Si}_{24} ;{ }^{48}$ it was later studied specifically with respect to its thermal properties, using DFT. ${ }^{49}$ Other metastable silicon allotropes have been observed in laser-induced transformations. ${ }^{50}$ An overview, including possible synthesis routes, was given recently.

In the present work, we explore how phonon properties for a diverse ensemble of crystal structures (allotropes or polymorphs) can be described in the GAP framework. We discuss computational protocols by which reference databases can be assembled, and potentials fitted, to deal with a range of silicon allotropes. Our work outlines a general strategy for generating GAP models that can be interfaced to high-throughput materials workflows.

\section{METHODOLOGY}

\section{A. Gaussian approximation potentials}

Interatomic forces, on which all of the present study is based, were obtained in the GAP framework. ${ }^{20}$ Initially, we tested the potential model for silicon developed by Bartók et al., ${ }^{30}$ which has been designed as a "general-purpose" interatomic potential for various applications in physics and materials science and extensively validated for physical properties of crystalline ${ }^{30}$ and amorphous ${ }^{52,53}$ silicon. We refer to this potential as "GAP-18" in the following. In the present work, we describe two methodological advances over that previous study. First, we develop new fitting databases by various strategies, with a specific view to describe vibrational properties, as detailed in Sec. III. Second, we use an atom-wise adaptive regularization scheme to improve the accuracy of the fit.

In brief, and using the notation of Ref. 30, the energy in GAP-18 is fitted as

$$
E=\sum_{i<j} V^{(2)}\left(r_{i j}\right)+\sum_{i} \varepsilon_{i},
$$

where the first sum is a baseline pair potential to capture exchange repulsion at short interatomic distances and the second sum is given by the Gaussian process regression itself. The atomic energy, $\varepsilon_{i}$, of a given atom, unknown from DFT but the key quantity in GAP, is

$$
\varepsilon_{i}=\sum_{s}^{M} \alpha_{s} K\left(\mathcal{R}_{i}, \mathcal{R}_{s}\right),
$$

based on a kernel (or similarity) function, $K$. The latter compares the local environment of the $i$-th atom in a given structure, $\mathcal{R}_{i}$, with all $M$ environments in a sparse reference set, $\mathcal{R}_{s}$. In GAP-18, this kernel function is given by the Smooth Overlap of Atomic Positions (SOAP) formalism; ${ }^{54}$ we use the same SOAP parameters as in Ref. 30 to ensure comparability.

The task in fitting a GAP model is therefore to find the regression coefficients, $\alpha$. Assume we have a reference database of $n$ structures, for each of which we know DFT-computed energies, $E_{j}$, and forces, $\mathbf{F}_{j, j^{\prime}}$, with scalar components in $x, y$, and $z$ directions for all $j^{\prime}$ atoms in the $j$-th structure. (We also add DFT-computed virial stresses, which do not change the approach but make the expressions more bulky, so we only discuss the case of energies and forces below.) We collect all entries of our DFT reference database in a single vector

$$
\mathbf{y}=\left(E_{1}, \ldots, E_{n}, F_{1,1}^{(x)}, \ldots, F_{n, j^{\prime}}^{(z)}\right) \text {. }
$$

We then define another vector, $\mathbf{y}^{\prime}$, which contains the atomic energies for all $N$ atoms in the reference database, which we do not know from DFT,

$$
\mathbf{y}^{\prime}=\left(\varepsilon_{1}, \ldots, \varepsilon_{N}\right) \text {. }
$$

We finally define a linear differential operator, $\mathbf{L}$, which connects the two,

$$
\mathbf{y}=\mathbf{L y}^{\prime}
$$

The fitting coefficients are then obtained from the reference data, encoded by $\mathbf{y}$, according to ${ }^{30,55}$

$$
\alpha^{*}=\left[\mathbf{K}_{M M}+\left(\mathbf{L} \mathbf{K}_{N M}\right)^{T} \boldsymbol{\Lambda}^{-1} \mathbf{L K}_{N M}\right]^{-1}\left(\mathbf{L} \mathbf{K}_{N M}\right)^{T} \boldsymbol{\Lambda}^{-1} \mathbf{y}
$$

with $\mathbf{K}$ denoting kernel matrices based on $K$ defined above (here, SOAP) and the size of $\mathbf{K}_{N M}$ being the number of total atoms, $N$, times the number of sparse points, $M$. The matrix $\Lambda$ is discussed in Subsection II B. We emphasize that in computational practice, neither $\mathbf{L}$ nor $\mathbf{K}_{N M}$ is computed individually; only, the combined matrix $\mathbf{L K}_{N M}$ is computed. To give an impression of the sizes of the matrices involved, the GAP-18 database contains $N=171,815$ atomic environments in total, of which $M=9000$ are selected as representative points.

The entries of $\mathbf{y}$ in the above equation (i.e., our DFT reference data) were obtained using CASTEP 8.0, ${ }^{56}$ with on-the-fly 
pseudopotentials, the PW91 functional, ${ }^{57}$ and a basis-set extrapolation scheme, ${ }^{58}$ and using the same convergence parameters as in GAP-18. ${ }^{30}$ All new potential versions were fitted with the same parameters as GAP-18 [including the baseline pair potential, $V^{(2)}$; Eq. (1)] but varied in the composition of the reference database and the number of sparse points, $M$. Parameter files for all new potential versions have been deposited in the Zenodo repository (see Data Availability statement).

\section{B. Regularization}

The key aspect of Eq. (6), in the context of the present work, is now the diagonal matrix $\Lambda$, which contains the expected errors for all entries of $y$,

$$
\boldsymbol{\Lambda}=\left(\begin{array}{cccccc}
\left(\sigma_{E}^{(1)}\right)^{2} & & & & & \\
& \ddots & & & & \\
& & \left(\sigma_{E}^{(n)}\right)^{2} & & & \\
& & & \left(\sigma_{F}^{(1)}\right)^{2} & \\
& & & & \ddots & \\
& & & & & \left(\sigma_{F}^{\left(n^{\prime}\right)}\right)^{2}
\end{array}\right) .
$$

In previous GAP fits, these expected errors have been set based on physical intuition and for separate parts of a given database (for example, assigning a smaller $\sigma_{E}$ to crystalline configurations and a larger $\sigma_{E}$ to amorphous ones). ${ }^{30,59}$ Instead, we now use a protocol for fitting phonon properties based on simple supercell displacements and an adjusted regularization (expected error) in the fit; a similar idea has been used very recently in Ref. 36 but not yet explored in detail. We here set the atom-wise regularization for force components in $\mathbf{F}_{i}=\left(F_{i}^{(x)}, F_{i}^{(y)}, F_{i}^{(z)}\right)$ according to

$$
\sigma_{F}^{(i)}= \begin{cases}f \times\left|\mathbf{F}_{i}\right|, & \text { if }\left|\mathbf{F}_{i}\right|>F_{\text {min }} \\ f \times F_{\min }, & \text { else. }\end{cases}
$$

Initially, we chose $f=0.1$ and $F_{\min }=0.01 \mathrm{eV} \AA^{-1}$. This value already leads to a lower bound of $\sigma_{\mathrm{F}}=0.001 \mathrm{eV} \AA^{-1}$, compared with $\sigma_{\mathrm{F}}=0.1 \mathrm{eV} \AA^{-1}$ for crystalline configurations in GAP- $18 .{ }^{30}$ We later vary $f$ over a wide range of even smaller values.

\section{Vibrational properties}

Phonon and thermal property computations were carried out using phonopy ${ }^{60}$ and phono3py ${ }^{6}$ with the finite displacement method. The size of the atomic displacements was $0.01 \AA$ (phonopy) and $0.03 \AA$ (phono3py), as per the standard setting. The thermal conductivity was calculated with the relaxation time approximation as implemented in phono3py.

To compute second-order and third-order force constants for optimized structures with the help of GAPs, a script was built that uses pymatgen 2019.12.22, ${ }^{61}$ Atomic Simulation Environment (ASE) $3.19 .0,{ }^{62}$ phonopy 2.4.2, phono3py 1.18.2, and quippy (including the GAP code, development version of 10 January 2020). The built-in geometry optimization of ASE was first used to optimize the crystal structures (including cell size) with the help of the GAPs. The forces on each atom were smaller than $10^{-5} \mathrm{eV} / \AA$. Phonon band structures were calculated along high-symmetry lines in reciprocal space; the latter were identified as described in Ref. 63 and as implemented in pymatgen. 51 points were calculated between each high-symmetry point. The supercells for the phonopy calculations were created based on the primitive cells in such a way that each lattice parameter was larger than $15 \AA$. To compare the DFT benchmark calculations of the harmonic phonons to GAPs, an rms value was defined that compares all bands along each point in reciprocal space at which the bands were calculated.

The supercells for the phono3py computations were based on the conventional cells, and they were also built such that each lattice parameter was $>15 \AA$. The thermal conductivity for diamond was calculated for the conventional cell and with an $11 \times 11 \times 11 q$-point grid. The complete code is available via Github (https://github.com/JaGeo/Phonons_GAPs_silicon) and archived in Zenodo (https://doi.org/10.5281/zenodo.3924470).

\section{RESULTS AND DISCUSSION}

\section{A. State of the art}

We begin by discussing the performance of GAP-18, ${ }^{30}$ a general-purpose ML potential. We quantify how well it can predict the phonon dispersion relations for diamond-type (dia) silicon, which is abundantly represented in its reference database (489 pristine and 404 defective bulk cells, some containing $>200$ atoms per cell, plus various surface configurations; Ref. 30) - but also for other allotropes that GAP-18 has not "seen." We compute all reference phonon band structures at the same level of DFT, enabling direct benchmarking. In the present work, we consider only structures at ambient pressure, but we mention that high-pressure silicon allotropes have been successfully studied with ML potentials before. ${ }^{64,65}$

For dia-Si [Fig. 1(a)], GAP-18 predicts phonons with practically quantitative accuracy, as previously shown in Ref. 30 . This is consistent with the results reported in Ref. 36, where a more specialized GAP model was fitted to snapshots from DFT-based molecular dynamics (MD) simulations of pristine and defective dia-Si. At this level of quality, the computed phonon band structures from both methods are practically indistinguishable to the naked eye. To quantify this agreement, we determined the root-mean-square (rms) error of phonon eigenvalues across the Brillouin zone, which amounted to $0.15 \mathrm{THz}$.

The situation is different for the clathrate-I-type structure, which is not included in the GAP-18 reference database [Fig. 1(b)]. Here, using the potential out of the box still leads to a dynamically stable structure (i.e., without any imaginary eigenvalues, which would conventionally be plotted as negative frequencies), and it does recover the general features. There are, however, notable quantitative differences, and some of them are highlighted in Fig. 1(b) by red circles. The highest-energy phonon mode at the zone center, $\Gamma$, is under-predicted by GAP by $0.8 \mathrm{THz}(6 \%)$, whereas the lowest predicted band at the $X$ point deviates by $0.3 \mathrm{THz}(12 \%)$ from the DFT reference data. In particular, the latter relative error is much larger (more than twice as large) than relative errors typically arising from DFT computations in comparison with experimental measurements: The mean relative error from DFT to the experiment calculated from 53 materials was $-3.6 \%$ in a recent high-throughput 

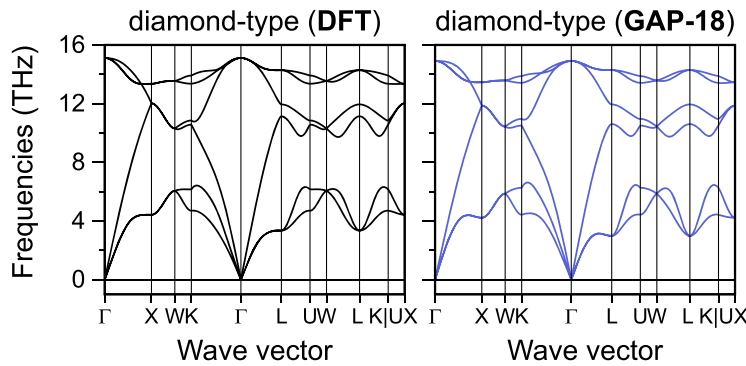

b
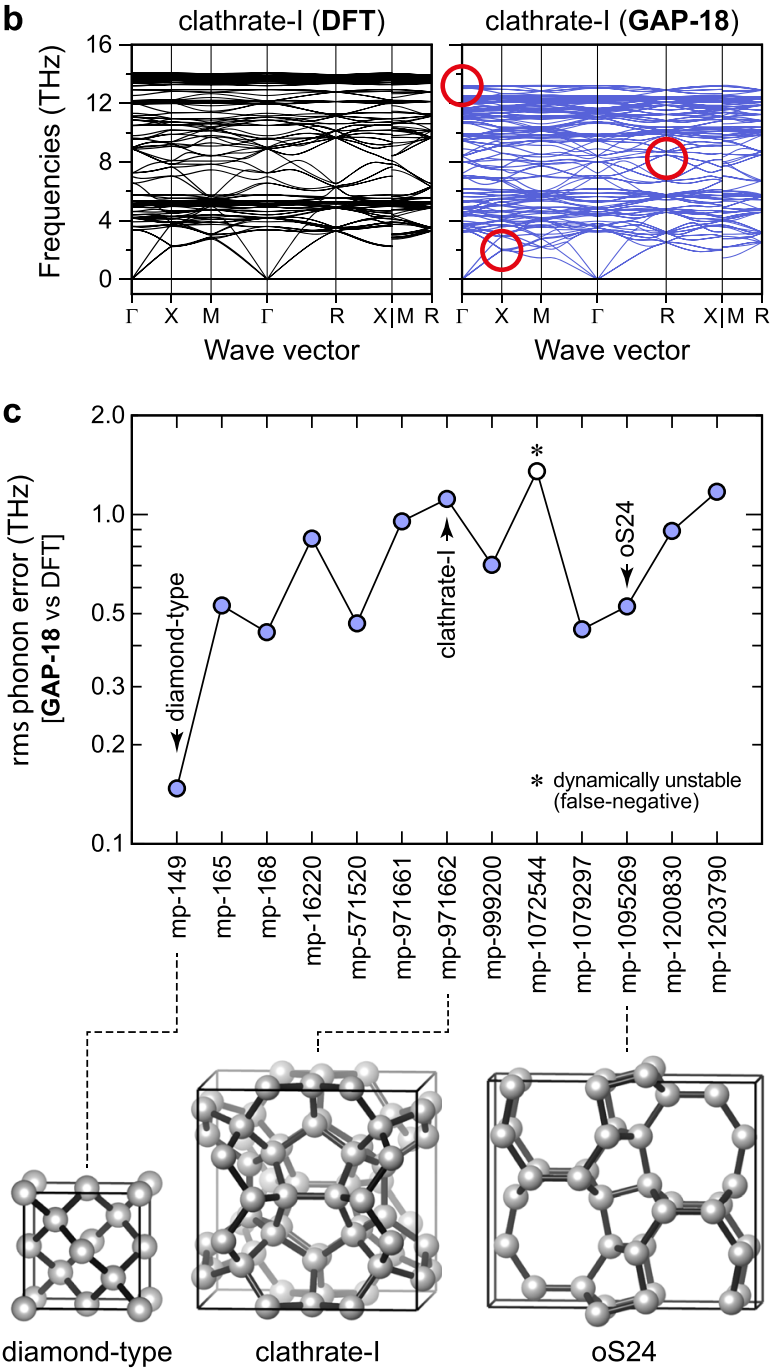

FIG. 1. Current state of the art in predicting phonons with the GAP methodology illustrated using the general-purpose GAP-18 model for silicon. ${ }^{30}$ (a) Phonon dispersion curves for the dia-type structure, computed with DFT (left) and GAP-18 (right), showing excellent agreement. (b) The same as (a) but for clathrate-l-type silicon. The general features of the phonon dispersion are reproduced as well, but there are several discrepancies in detail (highlighted in red), which are discussed in the text. (c) A survey of all ambient-pressure stable structures from the Materials Project database (using the "mp" identifier) up to a reasonable size and complexity and the root-mean-square error for their respective GAP-18 predicted phonon dispersions. Lines connecting data points are guides to the eye. study. Errors arising from ML potentials should therefore be in a similar range or smaller. The red circle at the $R$ point further highlights that the phonon frequencies calculated by GAP-18 are smaller than the ones calculated by DFT. The rms error is $1.11 \mathrm{THz}$, almost ten times that obtained for dia-Si.

To obtain a more comprehensive picture, we test the rootmean-square (rms) error of phonon eigenvalues for a wide range of reasonably sized and dynamically stable structures from the Materials Project database ${ }^{61}$ [Fig. 1(c)]. Out of 13 allotropes considered, all except one are correctly predicted to be dynamically stable. The exception is $\mathrm{mp}-1072544$, a hypothetical structure. ${ }^{42}$ The phonon prediction error for most allotropes is about $0.5 \mathrm{THz}-1.0 \mathrm{THz}$, with clathrate-I being one of the more poorly described examples. This is still a remarkable quality for structures that have not been included in the GAP-18 reference database, such as clathrate-I or oS24. ${ }^{48}$ However, the error is clearly too large for quantitative studies or for a fully reliable assessment of dynamic stability, which one might wish to carry out when computationally screening large amounts of hypothetical structures.

\section{B. Approaches for building fitting databases}

We now perform a comprehensive study of how reference databases can be designed for fitting phonon-accurate GAPs if no such prior database exists. Figure 2(a) provides an overview. We extract structures from the Materials Project database and filter them according to a maximum system size and the criterion that the structure has not significantly changed after the initial structural optimization with CASTEP. This structural change was determined by the StructureMatcher routine implemented in pymatgen, using slightly tighter tolerances for matching structures (ltol $=0.1$, stol $=0.1$, and angle_tol $=3$ ) instead of the default parameters. ${ }^{61} \mathrm{We}$ then create supercell models with atomic displacements and generate DFT reference data for those using two different strategies. A common approach uses randomly displaced cells, creating several copies with lattice parameters scaled by a few percent, and atomic positions randomized with a standard deviation of $0.01 \AA$, for example [green in Fig. 2(a)]. We also test a different approach (magenta): creating supercells of the optimized crystal structures with (only) individual displacements and atom-wise regularization, as discussed in Sec. II, for structures of reasonable size and complexity (up to 30 individual displacements). We finally fit candidate GAPs to the resulting databases, always using the same descriptors as in GAP18 , and evaluate their phonon errors by the same rms measure as in Fig. 1.

The randomized structures, as used in previous GAPs (e.g., Ref. 59), provide an acceptable but not a truly reliable result for the prediction of phonons [Fig. 2(b), top row]. We study the evolution of the error as a function of the number of sparse points, $M$, used in the fit. The outcome of this procedure varies notably from structure to structure; the prediction quality is better than that of GAP-18 for clathrate-I, about on par with that for the open-framework oS24 allotrope, but worse than that of GAP-18 for diamond-type silicon. This is indicative of the fact that a similar (and rather small) number of 20 distorted supercells is used to represent each structure, with a view to keep the computational workload tractable even for more complex chemical systems. The number of dia-like atomic environments in our databases is therefore much smaller than that in 


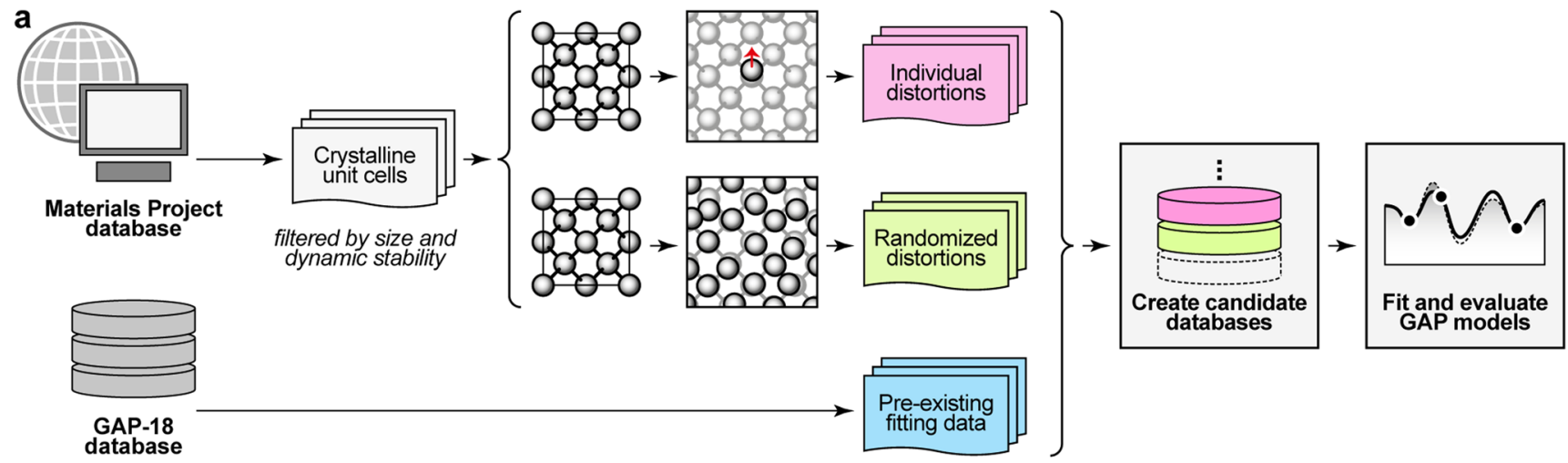

b
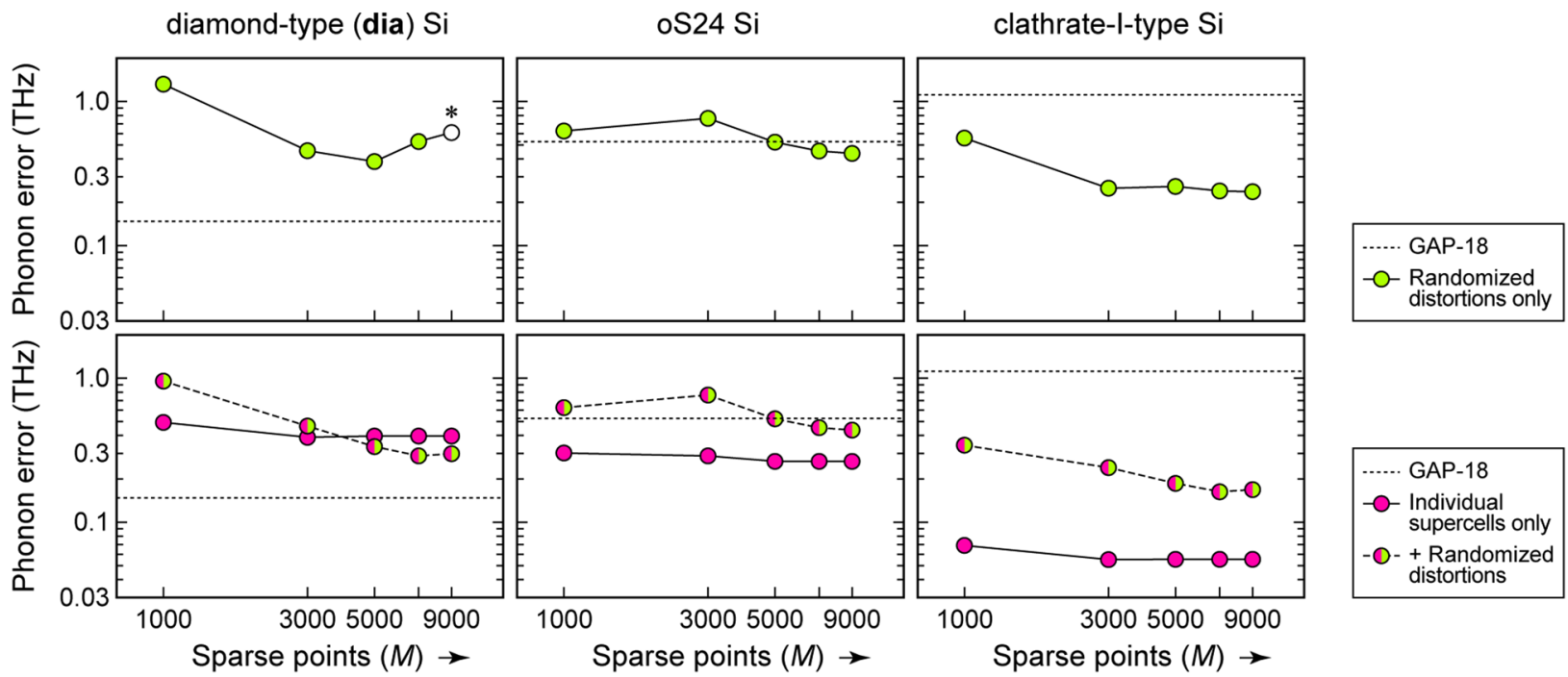

FIG. 2. (a) Strategies for building reference databases, shown by a simplified schematic. We extract crystal structures from the Materials Project database, with filtering as described in the text. We then create supercells based on these structures in which we displace either atoms individually or all of them randomly and compute DFT energies and forces for these supercells. Additionally, we investigate the effect of including the pre-existing GAP-18 database. ${ }^{30}$ We then build various combinations of databases to which candidate GAP models are fitted. (b) Results for various database building schemes sketched above, shown as phonon-error "learning curves" for three representative $\mathrm{Si}$ allotropes. An asterisk $(*)$ indicates a structure that is erroneously predicted as dynamically unstable. The reference values for GAP-18 are shown by dotted horizontal lines.

GAP-18. Interestingly, the dia "learning curve" does not converge with $M$ as expected, instead leading to a false-positive prediction of dynamic instability [“*” in Fig. 2(b)].

An alternative strategy is to create individual displacements (only), in separate supercells, akin to the way that one would build supercells for phonon computations. Displacements are generated along all symmetry-inequivalent directions, which leads to only one supercell for several structures including dia $(F d \overline{3} m)$, but many supercells for other structures, depending on space-group symmetry. In this case (magenta in Fig. 2), the phonon accuracy is generally better than that with randomized distortions only, and it plateaus after about 3000 representative atoms-this is clearly expected because the total number of relevant force-component entries in $\mathbf{y}$ [Eq. (3)] is relatively small, mostly relating to the displaced atom in a supercell and atoms in its local environment. There are, again, pronounced differences for the three allotropes, with clathrate-I again being most accurately described among those characterized in Fig. 2(b).

We also test combined models, which include Materials Project derived databases (and only those), indicated in Fig. 2(b) by mixed magenta/green symbols. This appears to even out the performance for the three different allotropes, increasing the phonon error for clathrate-I-type silicon, albeit not above $0.2 \mathrm{THz}$. The learning curves for the combined databases converge more slowly with $M$ than those for only individual displacements.

Having assessed the qualitative and individual performance of the method for selected structures, we now re-visit the full range 
of relevant silicon allotropes up to a reasonable size and complexity [as in Fig. 1 (c)], with results for rms phonon errors collected in Fig. 3. To make the comparison easier, we always choose $M=9000$, as in GAP-18.

Mirroring now more broadly what was already observed in Fig. 2(b), the prediction quality is overall quite scattered if only individual supercells are used to construct the reference database (top panel). For most of the silicon allotropes, the approach leads to a prediction error better than $0.5 \mathrm{THz}$; clathrate-I turns out to be the best described of all of them. When randomized distortions are used, on the other hand, the distribution of prediction errors is more uniform (green points in the bottom panel in Fig. 3). Two of the 13 structures are erroneously predicted to be dynamically unstable with both approaches-one of them being dia-Si in the case of using only randomized distortions, as noted above.

Adding individual supercells to the randomized distortions, i.e., combining both strategies outlined at the top of Fig. 2(a), appears to improve the results throughout. This is an important general finding: our individual-supercell strategy (magenta) can, and

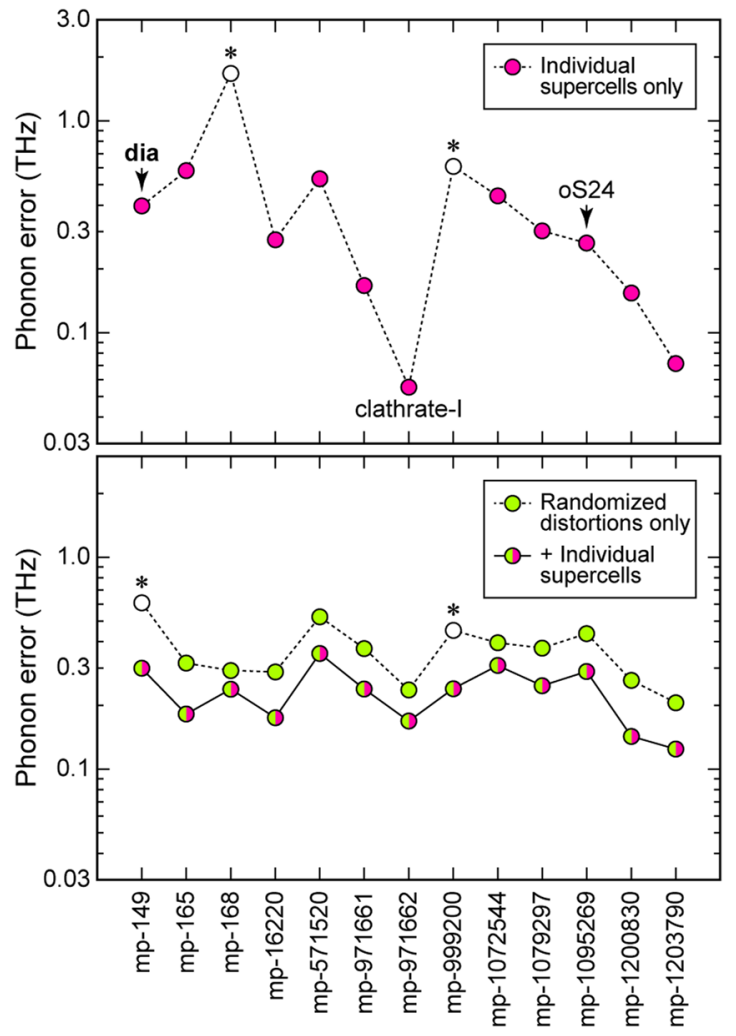

FIG. 3. Predicting phonon frequencies "from scratch" based on Materials Project entries. rms phonon band structure errors are given for all relevant mp entries as in Fig. 1(c), but now using new potentials, fitted to reference databases created as outlined in Fig. 2(a). All potentials use $M=9000$ sparse points. Top: results from the fit with only individual supercells. Two structures are erroneously predicted to be dynamically unstable $(*)$. Bottom: the same as top but for a potential fitted to randomly distorted structures only (green) and to a combined database (green/magenta). apparently should, be combined with other ways of sampling configuration space when developing GAP fitting databases. We emphasize that this protocol can be fully automated, requiring only a choice of the magnitude of the displacements and of the number of supercells to be created per structure; it is therefore expected to be easily coupled to existing and new high-throughput databases of crystal structures. An open research question, beyond the scope of the present work, is how these automated potentials can accommodate previously unseen structures that are not drawn from the Materials Project database or another database but are discovered, for example, during a GAP-driven random search. ${ }^{66}$

\section{Optimized regularization}

In this section, we analyze in more detail the effect of the atomwise regularization on the GAP prediction of phonon band structures. This corresponds to the idea of making the fit "looser" (more flexible) or "tighter" (more accurate in the required regions of configuration space, but therefore less flexible), controlled by the single factor $f$ that we use to scale all atom-wise force regularization parameters according to the absolute force on that atom [Eq. (8)]. We now perform GAP fits to versions of the "magenta" part of the database [Fig. 2(a)] in which we vary $f$ over several orders of magnitude and inspect its effect on the performance for dia, oS24, and clathrate-I-type silicon. We also test whether the additional inclusion of randomized distortions in the reference database would still improve the fit (as suggested by Fig. 3). We remind the reader that we had so far used $f=0.1$ throughout, and we now tighten the regularization, down to $f=0.0001$. These results are collected in Fig. 4.

For dia silicon, the phonon prediction error initially improves substantially when lowering $f$, viz., from about $0.4 \mathrm{THz}$ to about 0.2 THz. However, for $f=0.0001$, the error increases again, indicating a too low "expected error" for the input data. The quality of the fit can be even further improved, to better than $0.1 \mathrm{THz}$, if randomized distortions are added to the fitting database (note that we do not use atom-wise regularization for those configurations; instead, we employ the GAP-18 default of $\sigma_{F}=0.1 \mathrm{eV} \AA^{-1}$, to retain higher flexibility for more disordered configurations). For oS24, a qualitatively similar trend is observed in Fig. 4, although here the effect of too small $f$ is more drastic: the resulting potential led to a different structure during the relaxation that must be performed prior to evaluating the phonons, and therefore, no rms error for the latter can be obtained. This example more generally emphasizes the need for a (reasonably large) regularization of the GAP fit to arrive at stable results in practice. For clathrate-I-type silicon, the data shown in Fig. 4 similarly suggest the existence of an optimal $f$, and here, we observe an overall increase in phonon error when randomized configurations are added to the individually displaced supercellsa consequence of the "equalization" between different structures that had already been observed in Subsection III B. Taken together, these results suggest a choice of $f$ between 0.01 and 0.001 , but not smaller.

We note that the ongoing work in the community aims to optimize GAP fits with regard to the basis functions and hyperparameters for the structural descriptor. ${ }^{67-69}$ The present study complements these efforts by probing the question of how the regression task itself might be further optimized. 

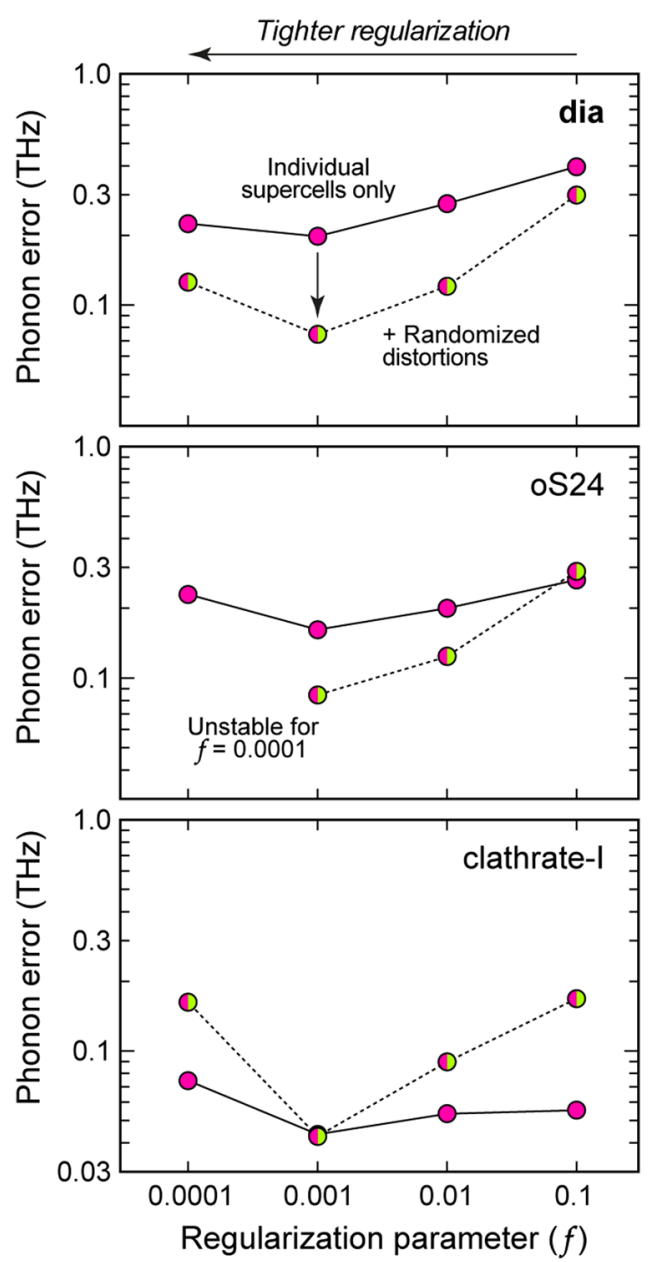

FIG. 4. Optimization of the regularization parameter $f$ as defined in Eq. (8). We performed series of fits, either with only the individually distorted Materials Project based supercells (magenta) or with the random displacements added (magenta/green). rms errors for computed vibrational eigenvalues are given as before. For oS24, the combined fit at $f=0.0001$ leads to a change in the structure during optimization, as a result of overfitting, and therefore, no rms error for the phonon band structure can be obtained. This indicates the reasonable limit within which $f$ should be chosen.

\section{Transferability: Extending GAP-18}

We now take our newly created reference databases, constructed from individual distortions with adaptive regularization [magenta in Fig. 2(a)] and randomized supercells [green in Fig. 2(a)], respectively, and combine them with the existing database to which the GAP-18 model had previously been fitted ${ }^{30}$ [blue in Fig. 2(a)]. We thereby aim to answer more general questions about GAPs: How can existing potentials be extended? How will these potentials retain their previously validated properties, viz., for GAP-18, the all-round accurate description of dia, liquid, and amorphous silicon?

Our combined fits use a total of $M=12000$ sparse points, of which 9000 are drawn from the previous database, with distribution among different configuration types (liquid, amorphous, etc.) as in GAP- $18,{ }^{30}$ and 3000 are drawn from a combined set of individually and randomly displaced supercells [Fig. 5(a)]. We combine both new databases because Fig. 3 indicates that doing this may lead to a more robust behavior than using the individually displaced cells on their own. Based on our analysis of the regularization parameter (Fig. 4), we generate two candidate potentials with $f=0.01$ and $f=0.001$, respectively.

Figure 5(b) shows that both potentials (with added supercells, "+SCs") reach a prediction quality within at least the $0.1 \mathrm{THz}-0.2$ $\mathrm{THz}$ range, with even better performance for some structures. There is a further minor improvement when moving from $f=0.01$ to

a
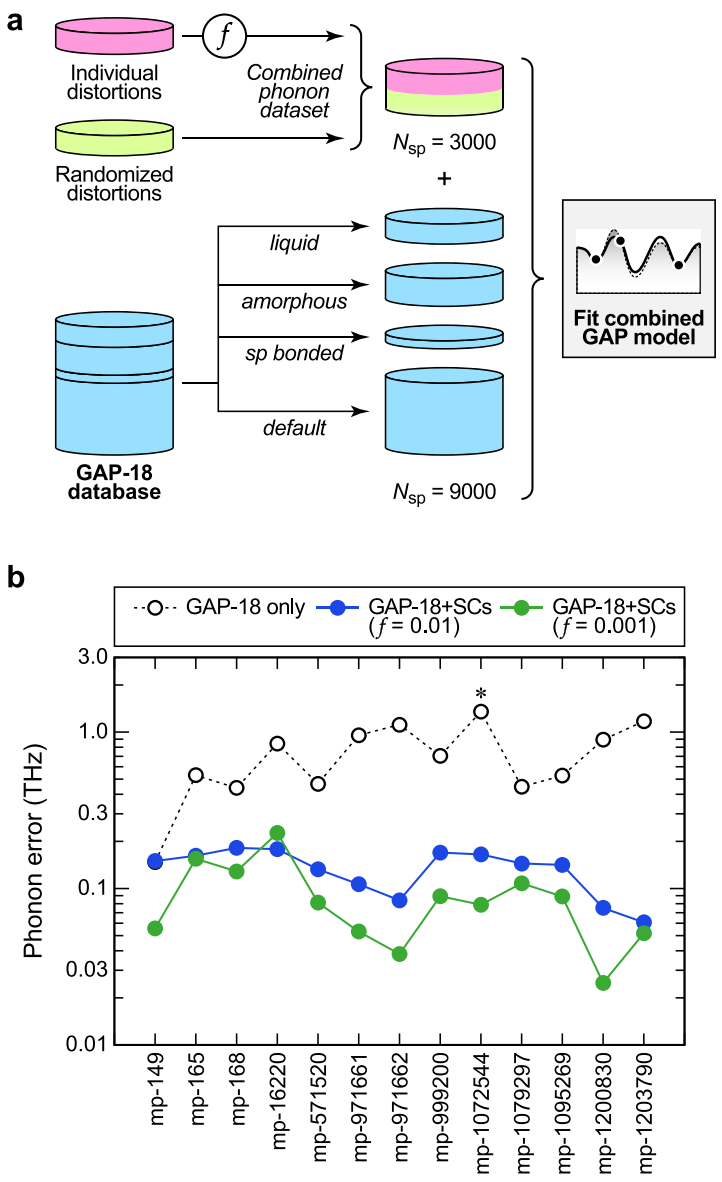

FIG. 5. (a) Building a reference database for the combined potential. Separate configuration type labels are set for the different parts of the GAP-18 database (blue), which are detailed in Ref. 30, and a new type is introduced for the datasets that we add in the present work (magenta/green). The number of sparse points, $N_{\mathrm{sp}}$, for each configuration type is given; their sum is the total number of sparse points, $M=12000$. (b) Prediction quality for phonons across the range of relevant structures-shown as in Fig. 3, but now comparing GAP-18 and extended potentials that are based on it. In both of these, the individually displaced and randomized supercells ("SCs") have been added to the fit, with atom-wise regularization according to $f=0.01$ and $f=0.001$ [cf. Eq. (8)], respectively. All structures are correctly predicted to be dynamically stable with both of the modified potentials. 
$f=0.001$, exemplified by an improved description of the dia structure with a smaller $f$, in line with what the tests in Fig. 4 have shown. However, both potentials would clearly seem useful for practical purposes when judged on the quality of their phonon predictions alone.

To test transferability of the newly created potentials, we compared the calculated thermal conductivity for dia with DFT references and experimental data. We emphasize that by "transferability," we mean that across structures, not across chemical compositions; our claims are strictly related to silicon. The thermal conductivity is a property that is reasonably well described by GAP-18, and the question is therefore whether our modifications lead to a detriment in that performance. To calculate accurate thermal conductivity, third-order force constants have to be calculated. Here, this is done with the help of a finite displacement method where a pair of atoms within the supercell has to be displaced. Such cells are not part of our reference data, and therefore, the potential needs to "pick up" the corresponding (anharmonic) physics from other cells in the database, such as MD snapshots. The previous GAP-18 model contains plenty of the latter, and therefore, it arrives at acceptable values for thermal conductivity in comparison with our DFT reference values (less than $15 \%$ deviation at each temperature, Table I). We mention in passing the good performance of independent, more specialized GAP and neural-network potential models for predicting $\kappa^{36-38}$

GAP-18+SCs $(f=0.01)$ shows the same results as the original GAP-18 within any reasonable accuracy, whereas $f=0.001$ leads to an entirely unreliable prediction of $\kappa$. This is now a crucial point because it indicates that the latter fit is too "tight" on the newly added structures (which correspond to harmonic phonons only) and, therefore, leads to overfitting behavior that manifests in poor prediction of forces in other regions of configuration space. It also emphasizes that the prediction quality for $\kappa$ is determined by the GAP-18 dataset. The Follow-up work on optimized databases with regard to predicting thermal conductivity and related properties is currently being planned.

We finally address the question whether the new potentials can also be used in the initial design space of GAP-18, for example, for disordered phases. GAP-18 has enabled accurate atomistic studies of liquid and amorphous silicon, ${ }^{52,53}$ and we therefore

TABLE I. Thermal conductivity, $\kappa$, for dia-Si as obtained from GAP-18 and extended versions of it, compared to DFT and experimental references at different temperatures.

\begin{tabular}{lrcr}
\hline \hline & \multicolumn{3}{c}{$\kappa\left(\mathrm{W} \mathrm{m}^{-1} \mathrm{~K}^{-1}\right)$} \\
\cline { 2 - 4 } Method & $300 \mathrm{~K}$ & $600 \mathrm{~K}$ & $900 \mathrm{~K}$ \\
\hline Expt. (Ref. 70) & 142.2 & 69.2 & 33.7 \\
\hline DFT (PBE, Ref. 49) & 137.4 & & \\
DFT (PW91, this work) & 126.4 & 57.1 & 37.4 \\
\hline GAP-18 & 110.1 & 50.4 & 33.1 \\
GAP-18+SCs $(f=0.01)$ & 110.2 & 50.5 & 33.1 \\
GAP-18+SCs $(f=0.001)$ & 53.7 & 26.5 & 17.7 \\
\hline \hline
\end{tabular}

investigate whether the extended versions are still applicable to the same problems. We designed a test that critically assesses the energy and force accuracy during GAP-driven melt-quench simulations. We do this by running an example trajectory that is representative of "real-world" applications, collecting an ensemble of structures, and

a

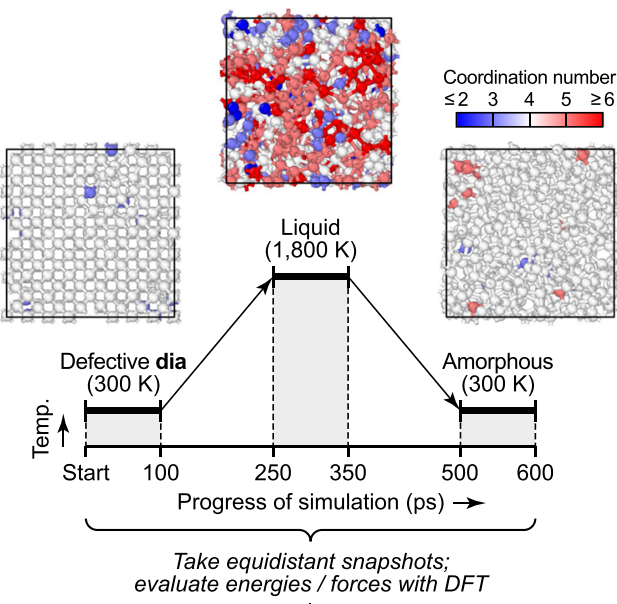

b

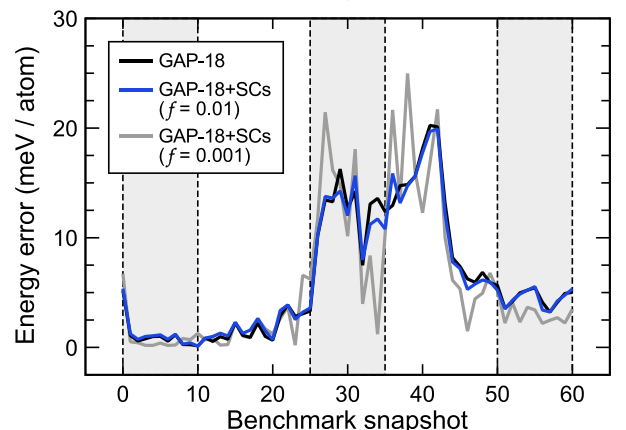

c

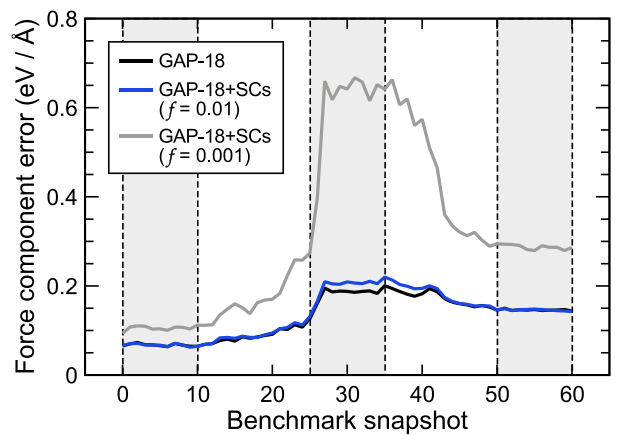

FIG. 6. Assessing the transferability of extended potentials by using a systematic benchmark for disordered structures. (a) Overview of the protocol: a defective diamond-type structure (500 atoms) is thermalized in constant-pressure moleculardynamics simulations driven by GAP-18, heated to $1800 \mathrm{~K}$, and cooled again (at $10^{13} \mathrm{~K} \mathrm{~s}^{-1}$ ) to form a quenched amorphous state, similar to Ref. 52. From this trajectory, we take structural snapshots every $10 \mathrm{ps}$ (10000 simulation steps) and evaluate their energies and forces with DFT. (b) Prediction error for the per-atom energy from various GAP models, given as absolute error vs DFT. (c) The same as (b) but now for the forces, with errors given as rms over all Cartesian force components in a given structure. 
benchmarking energies and forces along the trajectory against DFT data.

The starting point [Fig. 6(a)] is a 512-atom supercell of diamond-type silicon in which 12 atoms are randomly removed, leading to defects including a vacancy cluster. We run a GAPMD simulation over several hundreds of thousands of steps, in the NPT ensemble as implemented in the Large-scale Atomic/Molecular Massively Parallel Simulator (LAMMPS) software, ${ }^{71}$ similar to

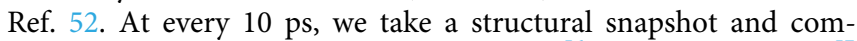
pute its energies and forces using CASTEP $^{56}$ at the PW91 level, ${ }^{5}$ using the same settings as those used for generating the GAP fitting data.

The energy error [Fig. 6(b)] is instructive to watch for the various new GAPs. It traces the GAP-18 result almost perfectly for $f=0.01$ but fluctuates much more strongly for $f=0.001$, already indicating that this model might not completely recover the behavior of the initial potential on which it is based. However, the absolute energy error is superficially not bad, and this has an important message for the benchmarking of GAPs: the average energy error itself might not provide sufficient information as to whether or not a given candidate potential is physically meaningful. Indeed, the averaged energy error appears to be best for $f=0.001$ in the final part of the liquid trajectory, viz., $8 \mathrm{meV}$ per atom compared to 12 for both other potentials (Table II). A strikingly different result, however, is seen in the force components [Fig. 6(c)], where $f=0.01$ recovers the behavior of GAP-18, whereas $f=0.001$ leads to more than triple the error.

This difference between the potentials not only manifests in different numerical errors, but it qualitatively affects whether they are in any way useful in practice. We repeated the diagnostic MD simulation as sketched in Fig. 6(a), but now using either of the extended potential models and otherwise similar settings. The results of this experiment are summarized in Fig. 7. The $f=0.01$ model led to a correct description of both the liquid and the quenched amorphous structures, indicated by radial distribution functions that are practically superimposable with results for the GAP-18 simulation [Fig. 7(b)]. In stark contrast, the potential fitted with $f=0.001$ led to

TABLE II. Numerical errors for the melt-quench test, as illustrated in Fig. 6(a), as a concise quality measure for the different potentials. Errors are averaged over the last five snapshots for each respective part of the trajectory.

\begin{tabular}{lccc}
\hline \hline & Defective dia & Liquid & Amorphous \\
\cline { 2 - 4 } & $(300 \mathrm{~K})$ & $(1800 \mathrm{~K})$ & $(300 \mathrm{~K})$ \\
\hline Energy error (meV/atom) & & \\
\hline GAP-18 & 0.5 & 12.2 & 4.3 \\
+SCs $(f=0.01)$ & 0.6 & 11.5 & 4.2 \\
+SCs $(f=0.001)$ & 0.6 & 8.4 & 2.6 \\
\hline Force component error $(\mathrm{eV} / \AA)$ & & \\
\hline GAP-18 & 0.07 & 0.19 & 0.15 \\
+SCs $(f=0.01)$ & 0.07 & 0.21 & 0.14 \\
+SCs $(f=0.001)$ & 0.11 & 0.65 & 0.29 \\
\hline \hline
\end{tabular}

a
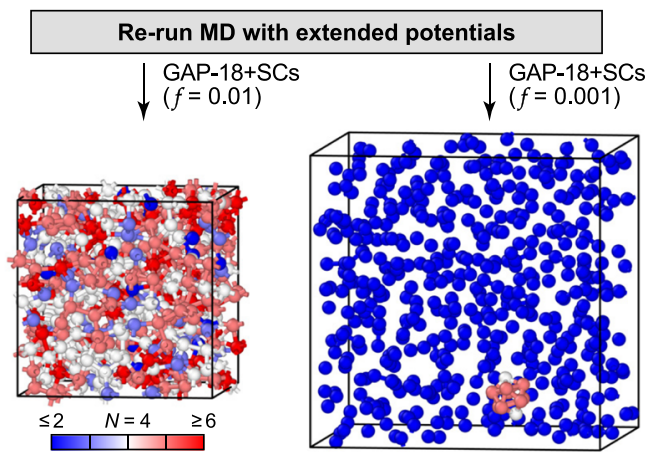

Correct description
of liquid retained

Unphysical result due to overfitting Analyze $\downarrow$ further

b

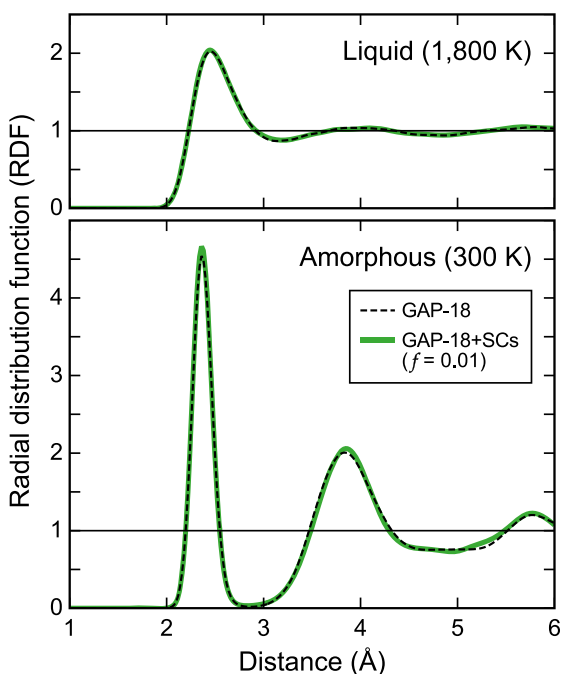

FIG. 7. Assessing the transferability of extended potentials by using them to run diagnostic MD simulations of liquid and amorphous silicon. (a) Snapshots after 300 ps of simulation time, which correspond to melted Si in the scheme of Fig. 6(a). The potential with $f=0.01$ reproduces the behavior of GAP-18, whereas that with $f=0.001$ leads to an unphysical result during heating, which then does not change substantially for the remainder of the simulation. Note that the simulations were done with the variable cell volume, and therefore, the structure on the righthand side has strongly (erroneously) expanded compared to the correct one on the left-hand side. (b) Computed radial distribution functions for the last $50 \mathrm{ps}$ of the liquid and amorphous parts of the trajectory, respectively; in both cases, the result from the extended GAP-18+SCs ( $f=0.01$ ) potential (solid green lines) is indistinguishable from that of GAP-18 (dashed black lines).

an abrupt dissociation of the structure during heating-resulting in a strongly under-coordinated structure, indicated in blue, with only one cluster of atoms, shown on the right-hand side of Fig. 7(a). This structure persisted during the entire simulation, with only minimal atomic motion even at $1800 \mathrm{~K}$. This exercise underlines the need for careful testing and validation of ML potentials in computational practice.

In summary, our extended potential with $f=0.01$ does not lead to any notable loss in quality for disordered silicon (Table II) 
while improving the description of phonons by almost an order of magnitude (Fig. 5).

\section{CONCLUSIONS}

The ability to compute accurate vibrational and thermal properties with ML potentials is an important result for two reasons. First, it has provided a useful testing ground for the way that reference databases for such potentials can be constructed with minimal computational effort-the main focus here being on the ability to treat various crystal structures at the same time. This may lead to a better understanding of the role and physical meaning of the "expected error" built into the GAP fit. Second, it is a step forward toward automated computational materials science, enabling the possibility of high-throughput vibrational property prediction especially when coupled to efficient workflows. ${ }^{72-74}$ It is expected that the approach outlined in the present work can be readily applied to other SOAP-GAP models and in fact extended to other fitting frameworks-essentially to any material that is amenable to an accurate ML potential fit. It is noted that these frameworks typically rely on the use of local structural descriptors and that ionic compounds with long-range Coulomb interactions might require additional methodological work. ${ }^{75,7}$

While most computational work for silicon to date has focused on the diamond-type allotrope, future searches for other structures and thereby synthesis targets are expected to be productive. The theory-guided discovery and, ultimately, experimental realization of new materials have now been achieved in many cases, ${ }^{77}$ and they might be accelerated by ML-driven phonon computations as presented here. For the specific example of group 14 elements, we mention the successful synthesis of clathrate-II-type germanium ${ }^{78}$ and also the wealth of intercalated clathrate compounds, ${ }^{79}$ which may now be addressed with a similar computational methodology.

\section{AUTHORS' CONTRIBUTIONS}

J.G. and V.L.D. initiated and coordinated the project. J.G. designed and implemented the workflows for phonon and thermal property analyses. G.C. and A.P.B. proposed the force-proportional atom-wise regularization. A.P.B. implemented it in the code. G.C. provided advice on fitting. V.L.D. developed the reference databases and fitted the modified potentials. J.G. and V.L.D. wrote the paper with input from all authors.

\section{ACKNOWLEDGMENTS}

J.G. acknowledges support from the HPC-Europa3 programme (Grant No. INFRAIA-2016-1-730897), with the support of the EC Research Innovation Action under the H2020 Programme, and from the European Union's Horizon 2020 research and innovation programme with the Marie Skłodowska-Curie Grant under Agreement No. 837910. V.L.D. acknowledges a Leverhulme Early Career Fellowship. The authors are grateful for computational support from the UK National High Performance Computing Service, ARCHER, for which access was obtained via the UKCP consortium and funded by EPSRC (Grant Reference No. EP/P022561/1).
The authors would like to acknowledge the use of the University of Oxford Advanced Research Computing (ARC) facility in carrying out this work (https://doi.org/10.5281/zenodo.22558). The authors also acknowledge Consortium des Équipements de Calcul Intensif en Fédération Wallonie Bruxelles (CÉCI) for computational resources. Structural drawings were created using VESTA ${ }^{80}$ and OVITO. ${ }^{81}$ The authors thank Atsushi Togo for helpful input.

A.P.B. and G.C. are listed as inventors on a patent filed by Cambridge Enterprise Ltd. related to SOAP and GAP (US patent 8843509, filed on 5 June 2009 and published on 23 September 2014). The other authors declare no conflicts of interest.

\section{DATA AVAILABILITY}

The data that support the findings of this study are openly available in the Zenodo repository (https://doi.org/10.5281/zenodo. 3924402).

\section{REFERENCES}

${ }^{1}$ G. J. Snyder and E. S. Toberer, Nat. Mater. 7, 105 (2008).

${ }^{2}$ P. Carrier, R. Wentzcovitch, and J. Tsuchiya, Phys. Rev. B 76, 064116 (2007).

${ }^{3}$ R. P. Stoffel, C. Wessel, M.-W. Lumey, and R. Dronskowski, Angew. Chem., Int. Ed. 49, 5242 (2010).

${ }^{4}$ O. Hellman, P. Steneteg, I. A. Abrikosov, and S. I. Simak, Phys. Rev. B 87, 104111 (2013).

${ }^{5}$ J. M. Skelton, S. C. Parker, A. Togo, I. Tanaka, and A. Walsh, Phys. Rev. B 89, 205203 (2014).

${ }^{6}$ A. Togo, L. Chaput, and I. Tanaka, Phys. Rev. B 91, 094306 (2015).

${ }^{7}$ K. Mizokami, A. Togo, and I. Tanaka, Phys. Rev. B 97, 224306 (2018).

${ }^{8}$ G. Petretto, S. Dwaraknath, H. P. Miranda, D. Winston, M. Giantomassi, M. J. Van Setten, X. Gonze, K. A. Persson, G. Hautier, and G.-M. Rignanese, Sci. Data 5, 180065 (2018).

${ }^{9}$ See http://phonondb.mtl.kyoto-u.ac.jp/ for an online database of phonon computations.

${ }^{10}$ C. Toher, J. J. Plata, O. Levy, M. de Jong, M. Asta, M. B. Nardelli, and S. Curtarolo, Phys. Rev. B 90, 174107 (2014).

${ }^{11}$ A. Seko, A. Togo, H. Hayashi, K. Tsuda, L. Chaput, and I. Tanaka, Phys. Rev. Lett. 115, 205901 (2015).

${ }^{12}$ J. Behler, Angew. Chem., Int. Ed. 56, 12828 (2017).

${ }^{13}$ V. L. Deringer, M. A. Caro, and G. Csányi, Adv. Mater. 31, 1902765 (2019).

${ }^{14}$ Y. Zuo, C. Chen, X. Li, Z. Deng, Y. Chen, J. Behler, G. Csányi, A. V. Shapeev, A. P. Thompson, M. A. Wood, and S. P. Ong, J. Phys. Chem. A 124, 731 (2020).

${ }^{15}$ T. Mueller, A. Hernandez, and C. Wang, J. Chem. Phys. 152, 050902 (2020).

${ }^{16}$ J. Behler and M. Parrinello, Phys. Rev. Lett. 98, 146401 (2007).

${ }^{17}$ N. Artrith and J. Behler, Phys. Rev. B 85, 045439 (2012).

${ }^{18}$ J. S. Smith, O. Isayev, and A. E. Roitberg, Chem. Sci. 8, 3192 (2017).

${ }^{19}$ L. Zhang, J. Han, H. Wang, R. Car, and W. E, Phys. Rev. Lett. 120, 143001 (2018).

${ }^{20}$ A. P. Bartók, M. C. Payne, R. Kondor, and G. Csányi, Phys. Rev. Lett. 104, 136403 (2010).

${ }^{21}$ T. D. Huan, R. Batra, J. Chapman, S. Krishnan, L. Chen, and R. Ramprasad, npj Comput. Mater. 3, 37 (2017).

${ }^{22}$ S. Chmiela, A. Tkatchenko, H. E. Sauceda, I. Poltavsky, K. T. Schütt, and K.-R. Müller, Sci. Adv. 3, el603015 (2017).

${ }^{23}$ A. P. Thompson, L. P. Swiler, C. R. Trott, S. M. Foiles, and G. J. Tucker, J. Comput. Phys. 285, 316 (2015).

${ }^{24}$ A. Seko, A. Takahashi, and I. Tanaka, Phys. Rev. B 92, 054113 (2015).

${ }^{25}$ A. V. Shapeev, Multiscale Model. Simul. 14, 1153 (2016). 
${ }^{26}$ H. Eshet, R. Z. Khaliullin, T. D. Kühne, J. Behler, and M. Parrinello, Phys. Rev. B 81, 184107 (2010).

${ }^{27}$ G. C. Sosso, D. Donadio, S. Caravati, J. Behler, and M. Bernasconi, Phys. Rev. B 86, 104301 (2012).

${ }^{28}$ G. C. Sosso, V. L. Deringer, S. R. Elliott, and G. Csányi, Mol. Sim. 44, 866 (2018).

${ }^{29}$ P. Rowe, G. Csányi, D. Alfè, and A. Michaelides, Phys. Rev. B 97, 054303 (2018).

${ }^{30}$ A. P. Bartók, J. Kermode, N. Bernstein, and G. Csányi, Phys. Rev. X 8, 041048 (2018).

${ }^{31}$ L. Zhang, D.-Y. Lin, H. Wang, R. Car, and W. E, Phys. Rev. Mater. 3, 023804 (2019).

${ }^{32}$ M. R. G. Marques, J. Wolff, C. Steigemann, and M. A. L. Marques, Phys. Chem. Chem. Phys. 21, 6506 (2019).

${ }^{33}$ X. Qian and R. Yang, Phys. Rev. B 98, 224108 (2018).

${ }^{34}$ P. Korotaev, I. Novoselov, A. Yanilkin, and A. Shapeev, Phys. Rev. B 100, 144308 (2019).

${ }^{35}$ V. V. Ladygin, P. Yu. Korotaev, A. V. Yanilkin, and A. V. Shapeev, Comput. Mater. Sci. 172, 109333 (2020)

${ }^{36}$ H. Babaei, R. Guo, A. Hashemi, and S. Lee, Phys. Rev. Mater. 3, 074603 (2019).

${ }^{37}$ E. Minamitani, M. Ogura, and S. Watanabe, Appl. Phys. Express 12, 095001 (2019).

${ }^{38}$ X. Qian, S. Peng, X. Li, Y. Wei, and R. Yang, Mater. Today Phys. 10, 100140 (2019).

${ }^{39}$ X. Tang, J. Dong, P. Hutchins, O. Shebanova, J. Gryko, P. Barnes, J. K. Cockcroft, M. Vickers, and P. F. McMillan, Phys. Rev. B 74, 014109 (2006).

${ }^{40}$ A. J. Karttunen, T. F. Fässler, M. Linnolahti, and T. A. Pakkanen, Inorg. Chem. 50, $1733(2011)$.

${ }^{41}$ A. J. Karttunen and T. F. Fässler, ChemPhysChem 14, 1807 (2013).

${ }^{42}$ H.-Y. Zhao, J. Wang, Q.-M. Ma, and Y. Liu, Phys. Chem. Chem. Phys. 15, 17619 (2013).

${ }^{43}$ A. Mujica, C. J. Pickard, and R. J. Needs, Phys. Rev. B 91, 214104 (2015).

${ }^{44}$ M. Amsler, S. Botti, M. A. L. Marques, T. J. Lenosky, and S. Goedecker, Phys. Rev. B 92, 014101 (2015).

${ }^{45}$ V. A. Saleev, A. V. Shipilova, D. M. Proserpio, and G. Fadda, Eur. Phys. J. B 90, 150 (2017).

${ }^{46}$ L.-A. Jantke, S. Stegmaier, A. J. Karttunen, and T. F. Fässler, Chem. Eur. J. 23, 2734 (2017).

${ }^{47}$ C. He, X. Shi, S. J. Clark, J. Li, C. J. Pickard, T. Ouyang, C. Zhang, C. Tang, and J. Zhong, Phys. Rev. Lett. 121, 175701 (2018).

${ }^{48}$ D. Y. Kim, S. Stefanoski, O. O. Kurakevych, and T. A. Strobel, Nat. Mater. 14, 169 (2015).

${ }^{49}$ T. Ouyang, P. Zhang, H. Xiao, C. Tang, J. Li, C. He, and J. Zhong, J. Phys. D: Appl. Phys. 50, 425501 (2017).

${ }^{50}$ L. Rapp, B. Haberl, C. J. Pickard, J. E. Bradby, E. G. Gamaly, J. S. Williams, and A. V. Rode, Nat. Commun. 6, 7555 (2015).

${ }^{51}$ B. Haberl, T. A. Strobel, and J. E. Bradby, Appl. Phys. Rev. 3, 040808 (2016).

${ }^{52}$ V. L. Deringer, N. Bernstein, A. P. Bartók, M. J. Cliffe, R. N. Kerber, L. E. Marbella, C. P. Grey, S. R. Elliott, and G. Csányi, J. Phys. Chem. Lett. 9, 2879 (2018).
${ }^{53}$ N. Bernstein, B. Bhattarai, G. Csányi, D. A. Drabold, S. R. Elliott, and V. L. Deringer, Angew. Chem., Int. Ed. 58, 7057 (2019).

${ }^{54}$ A. P. Bartók, R. Kondor, and G. Csányi, Phys. Rev. B 87, 184115 (2013).

${ }^{55}$ J. Quiñonero Candela and C. E. Rasmussen, J. Mach. Learn. Res. 6, 1939 (2005); available at http://www.jmlr.org/papers/v6/quinonero-candela05a.html.

${ }^{56}$ S. J. Clark, M. D. Segall, C. J. Pickard, P. J. Hasnip, M. I. J. Probert, K. Refson, and M. C. Payne, Z. Kristallogr. - Cryst. Mater. 220, 567 (2005).

${ }^{57}$ J. P. Perdew, J. A. Chevary, S. H. Vosko, K. A. Jackson, M. R. Pederson, D. J. Singh, and C. Fiolhais, Phys. Rev. B 46, 6671 (1992).

${ }^{58}$ G. P. Francis and M. C. Payne, J. Phys.: Condens. Matter 2, 4395 (1990).

${ }^{59}$ V. L. Deringer and G. Csányi, Phys. Rev. B 95, 094203 (2017).

${ }^{60} \mathrm{~A}$. Togo and I. Tanaka, Scr. Mater. 108, 1 (2015).

${ }^{61}$ A. Jain, S. P. Ong, G. Hautier, W. Chen, W. D. Richards, S. Dacek, S. Cholia, D. Gunter, D. Skinner, G. Ceder et al., APL Mater. 1, 011002 (2013).

${ }^{62}$ A. H. Larsen, J. J. Mortensen, J. Blomqvist, I. E. Castelli, R. Christensen, M. Dułak, J. Friis, M. N. Groves, B. Hammer, C. Hargus et al., J. Phys.: Condens. Matter 29, 273002 (2017).

${ }^{63}$ W. Setyawan and S. Curtarolo, Comput. Mater. Sci. 49, 299 (2010).

${ }^{64}$ J. Behler, R. Martoňák, D. Donadio, and M. Parrinello, Phys. Rev. Lett. 100, 185501 (2008).

${ }^{65}$ J. Behler, R. Martoňák, D. Donadio, and M. Parrinello, Phys. Status Solidi B 245, 2618 (2008).

${ }^{66}$ N. Bernstein, G. Csányi, and V. L. Deringer, npj Comput. Mater. 5, 99 (2019).

${ }^{67}$ M. J. Willatt, F. Musil, and M. Ceriotti, Phys. Chem. Chem. Phys. 20, 29661 (2018).

${ }^{68}$ M. A. Caro, Phys. Rev. B 100, 024112 (2019).

${ }^{69}$ L. Himanen, M. O. J. Jäger, E. V. Morooka, F. Federici Canova, Y. S. Ranawat, D. Z. Gao, P. Rinke, and A. S. Foster, Comput. Phys. Commun. 247, 106949 (2020).

${ }^{70}$ H. R. Shanks, P. D. Maycock, P. H. Sidles, and G. C. Danielson, Phys. Rev. 130, 1743 (1963).

${ }^{71}$ S. Plimpton, J. Comput. Phys. 117, 1 (1995).

${ }^{72}$ S. P. Ong, W. D. Richards, A. Jain, G. Hautier, M. Kocher, S. Cholia, D. Gunter, V. L. Chevrier, K. A. Persson, and G. Ceder, Comput. Mater. Sci. 68, 314 (2013).

${ }^{73}$ G. Pizzi, A. Cepellotti, R. Sabatini, N. Marzari, and B. Kozinsky, Comput. Mater. Sci. 111, 218 (2016).

${ }^{74}$ K. Mathew, J. H. Montoya, A. Faghaninia, S. Dwarakanath, M. Aykol, H. Tang, I.-h. Chu, T. Smidt, B. Bocklund, M. Horton et al., Comput. Mater. Sci. 139, 140 (2017).

${ }^{75}$ N. Artrith, T. Morawietz, and J. Behler, Phys. Rev. B 83, 153101 (2011).

${ }^{76}$ S. A. Ghasemi, A. Hofstetter, S. Saha, and S. Goedecker, Phys. Rev. B 92, 045131 (2015).

${ }^{77}$ A. R. Oganov, C. J. Pickard, Q. Zhu, and R. J. Needs, Nat. Rev. Mater. 4, 331 (2019).

${ }^{78}$ A. M. Guloy, R. Ramlau, Z. Tang, W. Schnelle, M. Baitinger, and Y. Grin, Nature 443, 320 (2006).

${ }^{79}$ M. Beekman and G. S. Nolas, J. Mater. Chem. 18, 842 (2008).

${ }^{80} \mathrm{~K}$. Momma and F. Izumi, J. Appl. Crystallogr. 44, 1272 (2011).

${ }^{81}$ A. Stukowski, Modell. Simul. Mater. Sci. Eng. 18, 015012 (2010). 